\title{
Model of Cellular Mechanotransduction via Actin Stress Fibers
}

\author{
Cecile LM Gouget · Yongyun Hwang · Abdul I Barakat
}

\begin{abstract}
Mechanical stresses due to blood flow regulate vascular endothelial cell structure and function and play a key role in arterial physiology and pathology. In particular, the development of atherosclerosis has been shown to correlate with regions of disturbed blood flow where endothelial cells are round and have a randomly organized cytoskeleton. Thus, deciphering the relation between the mechanical environment, cell structure and cell function is a key step towards understanding the early development of atherosclerosis. Recent experiments have demonstrated very rapid $(\sim 100$ msec) and long-distance $(\sim 10 \mu \mathrm{m})$ cellular mechanotransduction in which prestressed actin stress fibers play a critical role. Here, we develop a model of mechanical signal transmission within a cell by describing strains in a network of prestressed viscoelastic stress fibers following the application of a force to the cell surface. We find force transmission dynamics that are consistent with experimental results. We also show that the extent of stress fiber alignment and the direction of the applied force relative to this alignment are key determinants of the efficiency of mechanical signal transmission. These results are consistent with the link observed experimentally between cytoskeletal organiza-
\end{abstract}

\section{Cecile LM Gouget}

Hydrodynamics Laboratory (LadHyX), Department of Mechanics, Ecole Polytechnique, CNRS UMR7646, Palaiseau, France

Yongyun Hwang

Faculty of Engineering, Department of Aeronautics, Imperial College, London, UK

Abdul I Barakat

Hydrodynamics Laboratory (LadHyX), Department of Mechanics, Ecole Polytechnique, CNRS UMR7646, Palaiseau, France

Tel.: +33169335268

Fax: +33169335292

E-mail: abdul.barakat@ladhyx.polytechnique.fr tion, mechanical stress, and cellular responsiveness to stress. Based on these results, we suggest that mechanical strain of actin stress fibers under force constitutes a key link in the mechanotransduction chain.

Keywords Mechanotransduction · Stress Fibers . Cytoskeleton · Endothelial Cells · Mechanical Model · Force Transmission

\section{Introduction}

Vascular endothelial cells, the cells that line the inner walls of blood vessels, are constantly subjected to mechanical stresses due to blood flow. These stresses regulate many aspects of cell structure and function and play a role in the development of atherosclerosis (Hahn and Schwartz, 2009; Davies, 2008; Chien, 2007). In arterial regions of branching and bifurcation where blood flow is disturbed, endothelial cells are generally round with isotropic cytoskeletal organization (Malek et al, 1999; Flaherty et al, 1972; Wong et al, 1983), and they exhibit a pro-inflammatory phenotype that is susceptible to atherosclerosis (Chatzizisis et al, 2007; Caro et al, 1969). In contrast, in regions of undisturbed flow, endothelial cells are elongated and exhibit cellular alignment and cytoskeletal polarization in the primary flow direction (Malek et al, 1999; Flaherty et al, 1972; Wong et al, 1983). These cells are also associated with an anti-inflammatory and atheroprotective phenotype (Davies, 2008). These two different endothelial cell phenotypes can be reproduced in vitro by subjecting the cells to either low or reversing shear stress (a form of disturbed flow) or to high and non-reversing shear stress (representative of undisturbed flow) (Dewey et al, 1981; Helmlinger et al, 1991; Galbraith et al, 1998; Chatzizisis et al, 2007). Despite these observations, the relationship between flow-derived mechanical stresses, en- 
dothelial cell cytoskeletal organization (or cell shape), and cellular susceptibility to atherosclerosis remains to be elucidated.

How cells respond to mechanical cues is a subject of intense research interest (Orr et al, 2006; Hoffman et al, 2011). Key questions include how cells sense mechanical stimuli, how mechanical signals are transmitted within cells, and how these signals ultimately regulate gene expression and protein synthesis. Several candidate mechanosensors have been identified including the cell membrane (Haidekker et al, 2000), the glycocalyx (Tarbell and Pahakis, 2006; Florian et al, 2003), mechanosensitive ion channels (Barakat et al, 2006; Sukharev et al, 2001), focal adhesion sites and associated proteins (Geiger et al, 2009; Choquet et al, 1997; Friedland et al, 2009), and cell-cell adhesion complexes (Leckband et al, 2011; Tzima et al, 2005; Yonemura et al, 2010). The principal mechanotransduction mechanisms proposed thus far involve mechano-chemical conversion by one of these structures and subsequent transmission of the resulting chemical signal to target intracellular sites via either reaction-diffusion cascades or molecular translocation. One issue, however, is that these processes are relatively slow. The largest reported diffusion coefficient of proteins in the cytoplasm is $\sim 60$ $\mu \mathrm{m}^{2} / \mathrm{s}$ (Costa et al, 2006), which yields a minimum diffusion time across a typical cell length $(20 \mu \mathrm{m})$ of $\sim 6$ sec. Translocation of proteins via molecular motors requires comparable transmission times of a few seconds (Ashkin et al, 1990). Recent experiments, however, have demonstrated that a force exerted on the cell surface can induce a biological response across the cell within $\sim 300 \mathrm{msec}$ ( $\mathrm{Na}$ et al, 2008; Poh et al, 2009), a time too short to be explained by either reaction-diffusion cascades or molecular translocation. More specifically, upon application of a force, there is very rapid activation of the mechanosensitive proteins $\mathrm{Src}(\mathrm{Na}$ et al, 2008) and Rac (Poh et al, 2009), dissociation of protein complexes in the nucleus (Poh et al, 2012), as well as displacement of cytoplasmic and nucleolar structures (Hu et al, 2003, 2005). The mechanisms by which very rapid mechanical signal transmission occurs within cells remain to be elucidated.

There is mounting evidence that very rapid longdistance mechanical signal transmission requires an intact cytoskeleton ( $\mathrm{Na}$ et al, 2008; Poh et al, 2009; Hu et al, 2005; Wang et al, 2009). A force applied to a network of cytoskeletal fibers is transmitted through the fibers at the elastic wave speed, on the order of 30 $\mathrm{m} / \mathrm{s}$ (Na et al, 2008). At this speed, the force would be transmitted across a cell in $\sim 1 \mu$ sec, a virtually instantaneous response compared to any of the time scales discussed above. So, the cytoskeleton provides a pathway for transmitting a mechanical signal virtually instantaneously throughout a cell. Furthermore, the observation that the binding of specific proteins to actin stress fibers depends on the extent of stretch of the fibers (Sawada and Sheetz, 2002; Colombelli et al, 2009) suggests that the role of the cytoskeleton in mechanotransduction may extend beyond rapid force transmission to direct mechano-chemical conversion.

Theoretical models, based on various approaches such as the shear lag model (Wang and Suo, 2005) and a modified continuous approach (Blumenfeld, 2006), have shown that non uniform long-distance propagation of forces can be mediated by deformation of cytoskeletal fibers, but these models do not address the dynamics associated with this propagation. In light of the experimental observation that rapid transmission of mechanical signals in cells depends specifically on prestressed actin stress fibers (Na et al, 2008), Hwang and Barakat proposed a model for mechanical signal transmission through a single, prestressed, viscoelastic stress fiber (Hwang and Barakat, 2012). They showed that when stress fiber viscoelasticity is taken into account, the time scale for stress fiber deformation can be on the order of 1-10 msec, approaching that observed experimentally. They also showed that fiber prestress leads to two very different time scales for signal transmission, depending on whether the force is applied in the longitudinal or transverse direction relative to the fiber, thus potentially allowing the cell to distinguish between these two different directions of force application.

In the present work, we extend the single-fiber model of Hwang and Barakat to study mechanical signal transmission through a system of several stress fibers. We wish to particularly study the dependence of mechanical signal transmission on the extent of stress fiber alignment in order to explore if different cytoskeletal configurations as seen in elongated versus round endothelial cells transmit mechanical signals differently. We hypothesize that the relevant parameter for mechanical signal transmission through the cytoskeleton is not the force, which is virtually instantaneously transmitted within the cell, but rather the force-induced strain, whose development is delayed due to the viscoelasticity of stress fibers. In this new paradigm, the mechanical signal would induce protein activation not through stress but rather through strain, which has already been proposed as a possible mechanism for protein activation (Sawada and Sheetz, 2002; Han et al, 2004). Our results demonstrate that strain- mediated mechanical signal transmission through actin stress fibers allows a cell to integrate information derived from both the nature of the applied external force and the organization of the stress fibers. These findings have in- 
a

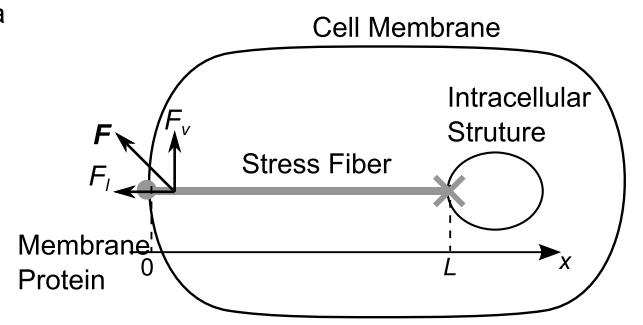

b

(i)

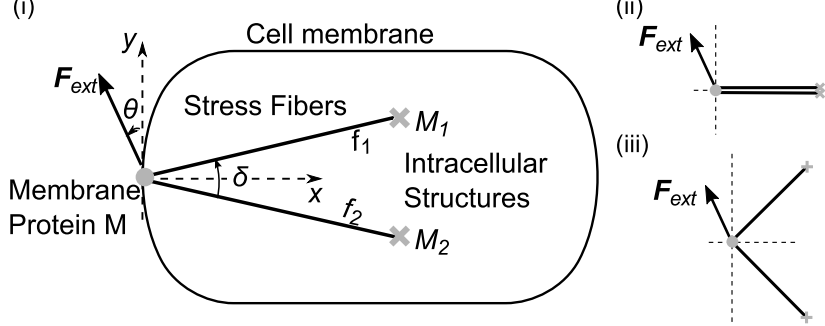

Fig. 1 a. Schematic of an actin stress fiber that directly connects a membrane protein such as an integrin or a focal adhesion at one end to an intracellular structure such as the nucleus at the other end. b. (i) The simplified two-fiber network in the context of the cell: the two fibers are linked at a moving node modeling a membrane protein (filled circle), and they connect to distinct intracellular structures (crosses) at the other end. (ii) Directionally aligned network $\left(\delta=0^{\circ}\right)$. (iii) Isotropically aligned network $\left(\delta=90^{\circ}\right)$

teresting implications for potential links among arterial flow-induced stresses on endothelial cells, endothelial cell shape, and endothelial cell phenotype.

\section{Methods}

\subsection{Simplified model of a single stress fiber}

Following the previous work (Hwang and Barakat, 2012; Hwang et al, 2012), we model actin stress fibers as uniformly prestressed viscoelastic filaments that link cell membrane proteins such as integrins/focal adhesions to other focal adhesions or to intracellular structures such as the nucleus. As depicted in Fig. 1a, we consider a stress fiber of length $L$, cross sectional area $A$, second moment of area $I$, density $\rho$, internal viscosity $\gamma$, elastic modulus $E$, and prestress $\sigma_{p}$. The stress fiber is surrounded by a cytoplasm of viscosity $\mu$ that resists fiber transverse and longitudinal motion with drag coefficients $C_{v}$ and $C_{l}$, respectively.

The single stress fiber model of Hwang and Barakat (2012) led to partial differential equations that describe stress fiber movement. Application of a force $F_{v}$ orthogonal to the stress fiber axis at a point not far from the membrane protein results in a transverse displacement $w_{v}$ governed by the following momentum balance, de- rived from the equilibrium of moments:

$$
\begin{aligned}
\rho A \frac{\partial^{2} w_{v}}{\partial t^{2}}= & \frac{\partial}{\partial x}\left(\sigma_{p} A \frac{\partial w_{v}}{\partial x}\right)- \\
& \frac{\partial}{\partial x}\left(E I \frac{\partial^{3} w_{v}}{\partial x^{3}}+\gamma I \frac{\partial^{4} w_{v}}{\partial x^{3} \partial t}\right)-C_{v} \mu \frac{\partial w_{v}}{\partial t}+F_{v}
\end{aligned}
$$

In this expression, stress fiber inertia is balanced by the restoring forces due to prestress $\sigma_{p}$ and flexural rigidity $E I$, the internal damping force due to the flexural material viscosity $\gamma I$, the cytosolic drag force, and the external force $F_{v}$. Note that the restoring force due to prestress is proportional to $\partial w_{v} / \partial x$, the bending moment in the beam is proportional to $\partial^{2} w_{v} / \partial x^{2}$, and the restoring force by bending rigidity is proportional to $\partial^{3} w_{v} / \partial x^{3}$. As in (Hwang and Barakat, 2012), we consider a stress-free boundary condition at the membrane protein and a pinched boundary condition at the other end of the stress fiber:

$$
\begin{aligned}
& \left.\frac{\partial w_{v}}{\partial x}\right|_{x=0}=\left.\frac{\partial^{3} w_{v}}{\partial x^{3}}\right|_{x=0}=0, \\
& \left.w_{v}\right|_{x=L}=\left.\frac{\partial^{2} w_{v}}{\partial x^{2}}\right|_{x=L}=0 .
\end{aligned}
$$

In the case of a longitudinal force $F_{l}$ along the stress fiber axis, the longitudinal displacement $w_{l}$ of the fiber is governed by the momentum balance:

$\rho A \frac{\partial^{2} w_{l}}{\partial t^{2}}=\frac{\partial}{\partial x}\left(E A \frac{\partial w_{l}}{\partial x}+\gamma A \frac{\partial^{2} w_{l}}{\partial x \partial t}\right)-C_{l} \mu \frac{\partial w_{l}}{\partial t}+F_{l}$.

Here, fiber inertia is balanced by the restoring force due to the elastic modulus $E$, the internal damping force due to the material viscosity $\gamma$, the cytosolic drag on the fiber, and the external force $F_{l}$. As in the case of transverse motion, we consider a stress-free boundary condition at the membrane protein and zero displacement at the the other end of the stress fiber:

$$
\begin{aligned}
& \left.\frac{\partial w_{l}}{\partial x}\right|_{x=0}=0, \\
& \left.w_{l}\right|_{x=L}=0 .
\end{aligned}
$$

For the case of multiple stress fibers that we wish to investigate here, it is desirable to explore possible simplifications of this model. In the previous work (Hwang and Barakat, 2012), it was shown that the bending stiffness is negligible compared to the stiffness due to prestress $\left(E I / L^{2} \sim 10^{-4} \sigma_{p} A\right)$ and that the dynamics of motion are predominantly determined by the fiber internal viscosity whereas the cytosolic drag and fiber inertia terms are negligible: 


$$
\begin{array}{r}
\tau_{v}^{\text {fiber }}=\frac{\gamma I / L^{2}}{\sigma_{p} A} \sim 10^{-4} \mathrm{~s} ; \tau_{v}^{\text {drag }}=\frac{C_{v} \mu}{\sigma_{p} A / L^{2}} \sim 10^{-5} \mathrm{~s} \\
\tau_{v}^{\text {inertia }}=\sqrt{\frac{\rho}{\sigma_{p}}} L \sim 10^{-6} \mathrm{~s},
\end{array}
$$

$$
\begin{aligned}
\tau_{l}^{f i b e r}=\frac{\gamma A}{E A} \sim 1 \mathrm{~s} ; & \tau_{l}^{v i s c}=\frac{C_{l} \mu}{E A / L^{2}} \sim 10^{-5} \mathrm{~s} \\
\tau_{l}^{\text {inertia }} & =\sqrt{\frac{\rho}{E}} L \sim 10^{-6} \mathrm{~s}
\end{aligned}
$$

where $\tau_{v}^{\text {fiber }}, \tau_{v}^{\text {drag }}$, and $\tau_{v}^{\text {inertia }}$ (respectively $\tau_{l}^{\text {fiber }}$, $\tau_{l}^{d r a g}$, and $\tau_{l}^{\text {inertia }}$ ) are the characteristic delay in transverse (respectively longitudinal) motion associated with internal fiber viscosity, cytosolic drag and inertia. It should be noted here that for the cytosolic drag term, we have considered cytoplasmic viscosities typically reported in the literature $\left(10^{-3} \mathrm{~Pa}\right.$-sec; see Table 1 below). Wang and Suo (2005) considered the potential additional contribution of surrounding cytoskeleton; this effect has not been taken into account in the present work.

Therefore, the equations of motion (1) and (2) can be simplified as follows:

$\sigma_{p} A \frac{\partial^{2} w_{v}}{\partial x^{2}}-\gamma I \frac{\partial^{5} w_{v}}{\partial x^{4} \partial t}+F_{v} \delta(x)=0$,

$E A \frac{\partial^{2} w_{l}}{\partial x^{2}}+\gamma A \frac{\partial^{3} w_{l}}{\partial x^{2} \partial t}+F_{l} \delta(x)=0$.

The fact that stress fiber inertia is negligible suggests that wave perturbations in the deformation field are damped by fiber internal viscosity. In support of this notion, the previous results for a single stress fiber (Hwang and Barakat, 2012) show that force transmission dynamics are indeed dominated by spatially monotonic deformation of stress fibers. Therefore, the structure of the deformation field does not change significantly in time, and further simplification (see the Appendix for details) yields:

$\frac{\sigma_{p} A}{L} w_{v}^{e n d}(t)+\frac{\gamma I}{L^{3}} \frac{d w_{v}^{e n d}(t)}{d t}=F_{v}$,

$\frac{E A}{L} w_{l}^{e n d}(t)+\frac{\gamma A}{L} \frac{d w_{l}^{e n d}(t)}{d t}=F_{l}$,

where $w_{v}^{e n d}$ and $w_{l}^{\text {end }}$ respectively denote the transverse and longitudinal displacements of the free end of the stress fiber. Thus, the single stress fiber can be modeled simply as a two dimensional anisotropic Kelvin-Voigt body, in agreement with recent experimental observations (Kumar et al, 2006). To examine the validity of the two ODEs (6a) and (6b), we compare the results obtained with these ODEs to the results obtained with the full PDE model (Hwang and Barakat, 2012). The ODE model predicts that when a steady transverse force $F_{v}$ is applied to the fiber, the resulting average strain of the fiber is:

$\epsilon_{v} \approx-\frac{w_{v}^{e n d}(t)}{L}=\frac{F_{v}}{\sigma_{p} A}\left(1-e^{-t / \tau_{v}}\right)$

where

$\tau_{v} \equiv \frac{\gamma}{\sigma_{p}}\left(\frac{I}{L^{2} A}\right)$

Similarly, the average strain under an axial force $F_{l}$ is:

$\epsilon_{l} \approx-\frac{w_{l}^{e n d}(t)}{L}=\frac{F_{l}}{E A}\left(1-e^{-t / \tau_{l}}\right)$,

where

$\tau_{l} \equiv \frac{E}{\gamma}$.

The time constants (7b) and (8b) are consistent with the time scales obtained by dimensional analysis in the previous work (Hwang and Barakat, 2012). Moreover, the time evolution of the mechanical signal dynamics described by (7a) and (8a) is remarkably similar to the dynamics described by the full PDEs, both for steady and oscillatory forces. The order of magnitude of the average strain is also well reproduced by the ODEs.

\subsection{The two-fiber system}

In this article, we focus primarily on a system of two stress fibers linking a membrane protein (moving node) to two intracellular sites (two fixed nodes) as represented in Fig. 1b, panel (i). This provides a simple model to study the role of cytoskeletal alignment, parametrized by the angle $\delta$ between the two fibers. As shown in Fig. 1b, when $\delta$ is small, the two fibers are aligned (panel (ii)), while when $\delta \approx 90^{\circ}$, there is no preferential direction, and the stress fiber organization is nearly isotropic (panel (iii)). In the context of arteries, the first case represents undisturbed flow regions of the arteries where endothelial stress fibers are highly aligned, whereas the second case describes disturbed flow regions where stress fibers are randomly oriented. Although the two-fiber system may appear very simple, we show in the last section of the Discussion that its behavior is indeed representative of that of more complex stress fiber networks. Thus, the two- fiber system can be viewed as the simplest stress fiber network 
that nonetheless captures the behavior of more complex networks that exist in cells.

Given the simplification that inertia and cytoplasmic drag are negligible, the following balance of forces must be enforced at the moving node $M$ :

$\mathbf{F}_{f 1 \rightarrow M}+\mathbf{F}_{f 2 \rightarrow M}+\mathbf{F}_{e x t}=\mathbf{0}$,

where $\mathbf{F}_{e x t}$ is the external force applied at node $M$, and $\mathbf{F}_{f_{i} \rightarrow M}$ is the force applied to $M$ due to the deformation of fiber $f_{i}$, where $i=1$ or 2 . We also note that due to the moment-free nature considered at the stationary nodes $M 1$ and $M 2$ (see equation (1)), the entire system is torque-free. Applying ODEs (6a) and (6b) to the fiber $f_{i}$ yields the components of $\mathbf{F}_{f_{i} \rightarrow M}$ transverse and longitudinal to the fiber, $F_{f_{i} \rightarrow M}^{v}$ and $F_{f_{i} \rightarrow M}^{l}$ :

$F_{f_{i} \rightarrow M}^{v}=\frac{\sigma_{p} A}{L}\left(w_{M_{i}}^{v}-w_{M}^{v}\right)+\frac{\gamma I}{L^{3}} \frac{d}{d t}\left(w_{M_{i}}^{v}-w_{M}^{v}\right)$

$F_{f_{i} \rightarrow M}^{l}=\frac{E A}{L}\left(w_{M_{i}}^{v}-w_{M}^{l}\right)+\frac{\gamma A}{L} \frac{d}{d t}\left(w_{M_{i}}^{v}-w_{M}^{l}\right)$,

where $w_{M}^{l}$ and $w_{M}^{v}$ are the displacements of node $M$ in the longitudinal and transverse fiber directions, respectively. Since the opposite node in each fiber is a fixed node, $\mathbf{w}_{M_{i}}=\mathbf{0}$ and the force depends only on $\mathbf{w}_{M}$. Substituting these equations into the balance of force (9) leads to the following system of linear differential equations of the motion of node $M$ :

$\boldsymbol{\Gamma} \frac{d \mathbf{w}_{M}}{d t}=-\mathbf{K w}_{M}+\mathbf{F}_{e x t}$,

where $\mathbf{K}$ and $\boldsymbol{\Gamma}$ are respectively the stiffness and damping matrices:

$\mathbf{K}=2\left[\begin{array}{cc}\frac{\sigma_{p} A}{L} \sin ^{2}\left(\frac{\delta}{2}\right)+\frac{E A}{L} \cos ^{2}\left(\frac{\delta}{2}\right) & 0 \\ 0 & \frac{\sigma_{p} A}{L} \sin ^{2}\left(\frac{\delta}{2}\right)+\frac{E A}{L} \cos ^{2}\left(\frac{\delta}{2}\right)\end{array}\right]$,

$\boldsymbol{\Gamma}=2\left[\begin{array}{cc}\frac{\gamma I}{L^{3}} \sin ^{2}(\delta)+\frac{\gamma A}{L} \cos ^{2}(\delta) & 0 \\ 0 & \frac{\gamma I}{L^{3}} \cos ^{2}(\delta)+\frac{\gamma A}{L} \sin ^{2}(\delta)\end{array}\right]$,

where $\delta$ is the angle between the two fibers.

Equations (11b) and (11c) show that the two axes of symmetry of the two fibers (the $\mathrm{x}$ - and $\mathrm{y}$ - axes) are the eigen directions of the system: only an external force applied along one of these axes will result in a displacement along the same direction as the force. In this case, equation (11a) can be analytically solved, and the displacement of the moving node is:

$\mathbf{w}_{M}=\left(\begin{array}{c}w_{M}^{x} \\ w_{M}^{y}\end{array}\right)=\left(\begin{array}{l}F_{e x t}^{x} / K_{x x}\left(1-\exp \left(-t / \tau_{x}\right)\right) \\ F_{e x t}^{y} / K_{y y}\left(1-\exp \left(-t / \tau_{y}\right)\right)\end{array}\right)$, where $\tau_{x} \equiv \Gamma_{x x} / K_{x x}, \tau_{y} \equiv \Gamma_{y y} / K_{y y}$ and $K_{x x}, K_{y y}$, $\Gamma_{x x}$, and $\Gamma_{y y}$ are the non-zero entries of the matrices $K$ and $\Gamma$ defined in (11b) and (11c). $\tau_{x}, \tau_{y}, K_{x x}$ and $K_{y y}$ are the characteristic times and stiffnesses of the system associated with the eigen directions.

\section{Results}

The model parameter values for the mechanical and geometric properties of stress fibers are derived from literature (Table 1).

Table 1 Values of mechanical and geometrical parameters.

\begin{tabular}{|c|c|c|}
\hline & Value & Source \\
\hline$R(\mathrm{~m})$ & $10^{-7}$ & $\begin{array}{l}\text { Deguchi et al } 2006 \\
\text { Kumar et al } 2006\end{array}$ \\
\hline$A\left(\mathrm{~m}^{2}\right)$ & $\pi 10^{-14}$ & $A=\pi R^{2}$ \\
\hline$I\left(\mathrm{~m}^{4}\right)$ & $\pi / 410^{-28}$ & $I=\int r^{2} d A$ \\
\hline$\rho\left(\mathrm{kg} / \mathrm{m}^{3}\right)$ & $10^{3}$ & $\mathrm{Na}$ et al 2008 \\
\hline$E(\mathrm{~Pa})$ & $10^{6}$ & $\begin{array}{l}\text { Deguchi et al } 2006 \\
\text { Lu et al } 2008\end{array}$ \\
\hline$\sigma_{p}(\mathrm{~Pa})$ & $310^{5}$ & Deguchi et al 2006 \\
\hline$\gamma(\mathrm{Pa} \mathrm{sec})$ & $410^{6}$ & Kumar et al 2006 \\
\hline$\mu(\mathrm{Pa} \mathrm{sec})$ & $10^{-3}$ & Hwang and Barakat 2012 \\
\hline$C_{v}$ & 1 & Hwang and Barakat 2012 \\
\hline$C_{l}$ & 0.8 & Hwang and Barakat 2012 \\
\hline
\end{tabular}

To determine the mechanical behavior of the twofiber system, we compute the effect of a known force on the stress fiber strain $\epsilon$ defined as:

$\boldsymbol{\epsilon}(x)=\frac{d \mathbf{w}_{M}}{d x}$.

The strain reduces to a vector because the stress fibers are modeled as one-dimensional beams whose sectional deformations are neglected.

As discussed in the Methods section, the strain is approximately uniform in $x$ along one fiber, so we will only consider the average strain in the fibers:

$\boldsymbol{\epsilon}=\frac{\mathbf{w}_{M}}{L}$,

where $\mathbf{w}_{M}$ is the displacement vector of the membrane protein. As shown in equations (11), the strain depends on the force direction $\theta=\arctan \left(-F_{\text {ext }}^{x} / F_{\text {ext }}^{y}\right)$ and on stress fiber alignment as specified by the angle $\delta$.

We consider an external force of magnitude $600 \mathrm{pN}$, which allows comparison of the model results to experimental results obtained using magnetocytometry. In the experiments, a stress of $\sim 20 \mathrm{~Pa}$ is applied to $4.5 \mu \mathrm{m}$-diameter beads bound to membrane integrins (Na et al, 2008; Hu et al, 2004; Wang and Ingber, 1994). The force applied on the bead is then $F_{\text {bead }} \approx 1270$ 


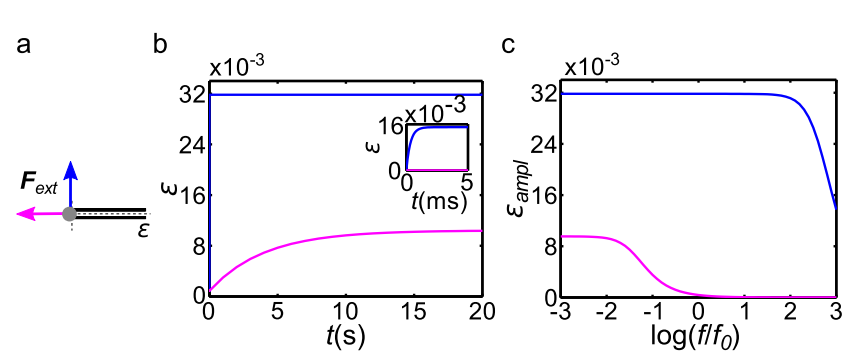

Fig. 2 Signal transmission dynamics in a network of two perfectly aligned stress fibers $\left(\delta=0^{\circ}\right)$ stimulated with an external force in either the transverse direction (blue) or the longitudinal direction (magenta). a. Schematic of the system: we apply an external force $\mathbf{F}_{\text {ext }}$ at the membrane end of the fibers, and we track the time evolution of the resulting strain in the fibers. b. Strain as a function of time when a step force of $600 \mathrm{pN}$ is applied. c Amplitude of strain versus log of frequency for a non-reversing oscillatory force $(\alpha=0.5$, $\left.\beta=0.75, F_{0}=600 \mathrm{pN}\right) . f_{0}$ is a reference frequency taken to be $1 \mathrm{~Hz}$

$\mathrm{pN}$. The adhesion density at the cell surface is typically $d=0.15$ adhesions $/ \mu \mathrm{m}^{2}$ (Davies et al, 1993, 1994). If 20 to $30 \%$ of the bead is embedded in the membrane, the bead surface area available for binding to membrane proteins is $0.8-1.2 \pi r^{2}$, where $r$ is the bead radius, and the number of cell-bead adhesions would be $\sim 0.8-1.2 \pi r^{2} d$. This yields a typical force per adhesion of $F_{\text {ext }}=F_{\text {bead }} / \pi r^{2} d \approx 600 \mathrm{pN}$. Varying the force amplitude does not change the qualitative behavior of the results as equation (11a) is linear, so that the computed strains scale directly with the magnitude of the applied force.

\subsection{Mechanical signal transmission in aligned fibers}

We begin by examining the case of two perfectly aligned stress fibers $\left(\delta=0^{\circ}\right)$ stimulated either along or orthogonal to the direction of fiber alignment as depicted schematically in Fig. 2a. In response to a constant force of $600 \mathrm{pN}$ applied as a step function, both the transverse and longitudinal strains reach a plateau after an initial transient phase (Fig. 2b). The steadystate transverse strain (plateau value) is approximately three times higher than the steady-state longitudinal strain, and it is attained in only a few milliseconds versus 10 to 20 seconds for the longitudinal strain. These differences in the magnitude of the strains and in the associated dynamics are attributable to the fact that stress fiber prestress is the primary determinant of fiber transverse movement, whereas fiber elasticity is the primary determinant of longitudinal movement $\left(\sigma_{p}=E / 3\right.$ (Table 1)) and are consistent with the previous work on a single stress fiber (Hwang and Barakat, 2012).
To more closely mimic physiological conditions in the arterial system, we examine the response of the two aligned fiber model to an oscillatory force of the form:

$F_{e x t}=F_{0}(\alpha \sin (2 \pi f t)+\beta)$,

where $f$ is the oscillation frequency, and $\alpha$ and $\beta$ are coefficients that modulate the amplitude, maximum, and mean of the applied force. In particular, if $\beta>\alpha>0$, the force does not change sign in time, characteristic of undisturbed flow zones, whereas if $\alpha>\beta>0$, the force changes sign periodically, a feature typical of disturbed flow regions.

We first consider a non-reversing oscillatory force ( $\alpha=0.5, \beta=0.75$ ), typical of undisturbed flow regions in arteries. Since the equations are linear, the resulting strain also oscillates with frequency $f$. Fig. 2c shows the amplitude of this strain $\epsilon_{a m p l}$ as a function of frequency, where $\epsilon_{a m p l}$ is defined as follows:

$\epsilon_{a m p l} \equiv \max _{\left[t_{0}, t_{0}+1 / f\right]}(\|\boldsymbol{\epsilon}(t)\|)-\min _{\left[t_{0}, t_{0}+1 / f\right]}(\|\boldsymbol{\epsilon}(t)\|)$,

where $t_{0}$ is sufficiently large for steady-state to be reached. These results demonstrate that the system of two aligned fibers is a low-pass filter whose cutoff frequency depends on the direction of force application. The cutoff frequency is significantly lower in the longitudinal direction than in the transverse direction. This difference in cutoff frequencies correlates with the time scale of the strain response to a step force (Fig. 2b). Interestingly, under physiological conditions, $f \approx 1 \mathrm{~Hz}$ (heart rate), the longitudinal signal is cut off whereas the transverse signal is not.

The results in Figs. $2 \mathrm{~b}$ and $2 \mathrm{c}$ show that application of either a step or oscillatory force to a system of two aligned stress fibers leads to drastically different signal transmission dynamics depending on whether the force is exerted along the axis or normal to the axis of fiber alignment. Interestingly, we obtain displacement values $w=\epsilon L$ of the order of $0.1 \mu \mathrm{m}$, in agreement with various experimental results ( $\mathrm{Hu}$ et al, 2004, 2003; Na et al, 2008).

3.2 Effect of fiber alignment on signal transmission efficiency

Fig. 3 illustrates the dependence of the steadystate longitudinal and transverse strains, $\epsilon_{a x}\left(t_{\infty}\right)$ and $\epsilon_{t r}\left(t_{\infty}\right)$ respectively, in the top fiber of the network on both fiber alignment $(\delta)$ and force direction $(\theta)$. Although only the strain values in the top fiber and for $\delta \in\left[0^{\circ}, 180^{\circ}\right]$ and $\theta \in\left[0^{\circ}, 180^{\circ}\right]$ are shown, the strains 

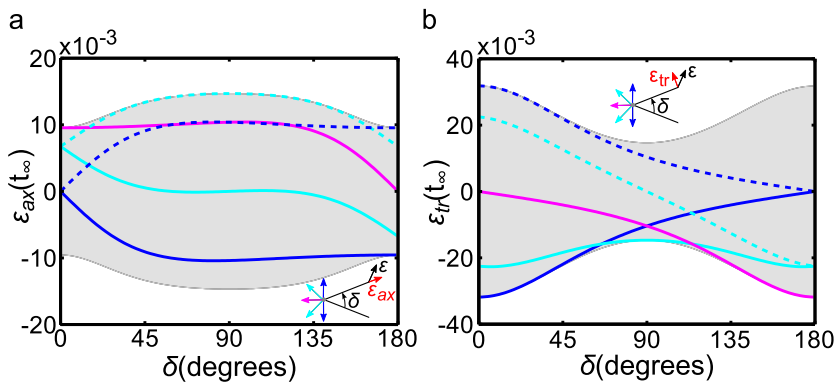

Fig. 3 Steady-state strain in the directions longitudinal (a) and transverse (b) to the top fiber as a function of the angle between the fibers, $\delta$, for different force directions - $\theta=0^{\circ}$ (solid blue line), $\theta=45^{\circ}$ (solid cyan line), $\theta=90^{\circ}$ (solid magenta line), $\theta=135^{\circ}$ (dashed cyan line), and $\theta=180^{\circ}$ (dashed blue line). The insets depict the configurations studied. The grey zones represent the envelope of values of the strain when the force direction spans the entire $\left[0^{\circ}, 360\right]$ interval. The steady-state strain is defined as the strain at $t \rightarrow \infty$ when a force of $600 \mathrm{pN}$ is applied to the fiber in a step manner

for all other $\delta$ and $\theta$ values and the strains in the bottom fiber can be readily deduced using symmetry arguments.

We consider that the steady-state strain in a stress fiber is a measure of the efficiency of mechanical signal transmission in that fiber. The results show that an external force applied in a given direction $\theta$ is transmitted with variable efficiency depending on the fiber alignment angle $\delta$. For instance, at $\theta=45^{\circ}$, the longitudinal strain in the top stress fiber is highly sensitive to fiber alignment and even changes sign, going from tension (positive strain) at small $\delta$ to compression (negative strain) at large $\delta$ (Fig. 3a). Fig. 3 also shows that for a given stress fiber alignment (fixed $\delta$ ), the transmitted strain ranges from compressive to tensile depending on the direction of the external force. The overall sensitivity of transmission efficiency to force direction is illustrated by the grey zones in Figs. $3 \mathrm{a}$ and $3 \mathrm{~b}$, which represent the envelope of strain values when the force direction spans the entire $\left[0^{\circ}, 360\right]$ range. The longitudinal strain (Fig. 3a) is most sensitive to force direction when the fiber organization is isotropic $\left(\delta=90^{\circ}\right)$, whereas transverse strain (Fig. $3 \mathrm{~b}$ ) is more sensitive to force direction when the fibers are aligned $\left(\delta=0^{\circ}\right.$ and $\left.\delta=180^{\circ}\right)$. These results show that the extent of stress fiber alignment regulates the efficiency of mechanical signal transmission and the sensitivity of the stress fibers to the direction of the externally applied force.
3.3 Effect of fiber alignment on signal transmission dynamics

Experiments suggest that actin stress fibers mediate very rapid transmission of mechanical signals within cells ( $\mathrm{Na}$ et al, 2008). We wish to explore how stress fiber alignment modulates the dynamics of mechanical signal transmission and how this modulation is affected by the direction of the externally applied force. To this end, we compute the characteristic time for strain development in the two-fiber network in both the longitudinal and transverse directions as a function of both the stress fiber alignment angle $\delta$ and the angle of force application $\theta$ (Fig. 4). For a constant force applied as a step function, the characteristic response time $T$ is defined such that:

$\epsilon(T) \leq \frac{1}{\sqrt{2}} \max _{t}(\epsilon(t))$.

For an oscillatory force, $T$ is defined as the cutoff period such that:

$A\left(\epsilon\left(F_{T}\right)\right) \leq \frac{1}{\sqrt{2}} \max _{\tau}\left(A\left(\epsilon\left(F_{\tau}\right)\right)\right)$,

where $F_{\tau}$ is a force of frequency $1 / \tau$ as defined by equation (18) and $A$ is the amplitude. The cutoff period and the characteristic response time are equivalent as they both characterize the dynamics of the system. Note that when fibers are aligned $\left(\delta=0^{\circ}\right.$ or $\left.\delta=180^{\circ}\right)$ and a force is applied along the direction of alignment $\left(\theta=90^{\circ}\right.$ or $\theta=0^{\circ}$ respectively), $\epsilon_{t r}=0$ and the characteristic time cannot be defined. Similarly, when the fibers are aligned $\left(\delta=0^{\circ}\right.$ or $\left.\delta=180^{\circ}\right)$ and a force is applied orthogonal to the direction of alignment $\left(\theta=0^{\circ}\right.$ or $\theta=90^{\circ}$ respectively), $\epsilon_{a x}=0$ and the corresponding characteristic time cannot be defined. As in Fig. 3, we limit the study to the top fiber and to $\delta \in\left[0^{\circ}, 180^{\circ}\right]$ and $\theta \in\left[0^{\circ}, 180^{\circ}\right]$. Information on the bottom fiber and all other $\delta$ and $\theta$ values can be obtained from symmetry considerations.

Fig. 4a indicates that a longitudinal strain can only be transmitted rapidly (grey zone) in the case of highly aligned configurations $\left(\delta \approx 0^{\circ}\right.$ or $\left.\delta \approx 180^{\circ}\right)$ stimulated by a force acting normal to the stress fibers. Fiber alignment is also necessary for rapid transmission of transverse strains (Fig. 4b); however, a broader range of force directions allows these dynamics. As suggested by the stiffness and damping matrices (11b) and (11c), the $\mathrm{x}-$ and $y$ - axes (axes of symmetry of the fibers) are eigen directions of the system, associated with two characteristic times whose interplay drives the dynamics of the system. Fig. 4 shows that in the case of two aligned fibers, the time scales for signal transmission along the two eigen directions are very different with $\tau_{l}=\tau_{x} \approx 5$ 

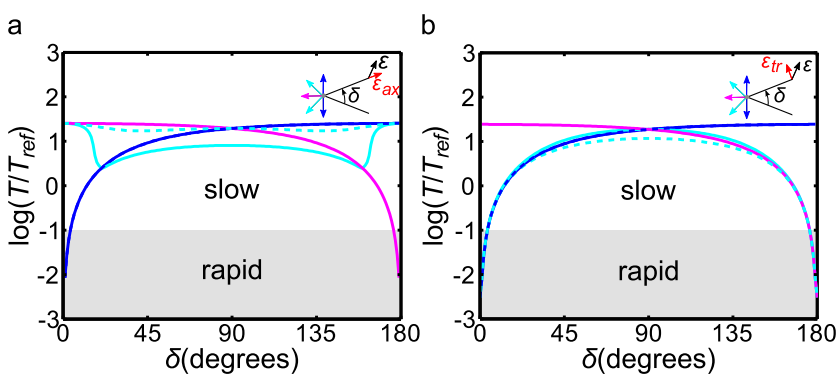

Fig. 4 Characteristic time of strain dynamics in the directions longitudinal (a) and transverse (b) to the top fiber as a function of the angle between the fibers, $\delta$, for different force directions - $\theta=0^{\circ}$ (solid blue line), $\theta=45^{\circ}$ (solid cyan line), $\theta=90^{\circ}$ (magenta solid line), $\theta=135^{\circ}$ (dashed cyan line) and $\theta=180^{\circ}$ (dashed blue line). The insets depict the configurations studied. $T_{r e f}$ is a reference period, $T_{r e f}=1 \mathrm{sec}$. The grey zones delimit the regions of rapid force transmission, taken as $0.1 T_{\text {ref }}(100 \mathrm{msec})$

sec and $\tau_{v}=\tau_{y} \approx 1$ msec. Thus, longitudinal strain dynamics are dominated by a slow time scale for all forces that have a non-negligible component in the longitudinal direction, and only the curves $\theta=0^{\circ}$ for $\delta=0^{\circ}$ and $\theta=90^{\circ}$ for $\delta=180^{\circ}$ are in the grey zone. On the other hand, the transverse strain is associated with rapid dynamics, and a broader range of external force directions can be rapidly transmitted. When the angle $\delta$ between the two fibers increases, the difference between the time scales decreases, and at $\delta=90^{\circ}$, the two time scales are equal with $\tau_{x}=\tau_{y} \approx 1 \mathrm{sec}$, and the overall dynamics of the system are slow.

Thus, the results in Fig. 4 suggest that rapid mechanical signal transmission is only possible when fibers are significantly aligned. Furthermore, the narrow range of force directions inducing rapid longitudinal strain suggests that longitudinal strain is not a robust mediator of rapid mechanical signal transmission.

\subsection{Spatial distribution of an applied force - strain} differences between fibers

We have thus far presented results only for the top fiber, since the results for the bottom fiber can be deduced from symmetry arguments. However, it is instructive to compare signal transmission through the top and bottom fibers in order to develop an appreciation for the spatial distribution of an applied force. To this end, we study the ratio of the steady-state longitudinal strain in the top fiber to that in the bottom fiber, $r_{a x}$, and the equivalent ratio for transverse strain, $r_{t r}$, i.e.:

$r_{a x}=\frac{\epsilon_{a x}^{u p}\left(t_{\infty}\right)}{\epsilon_{a x}^{\text {down }}\left(t_{\infty}\right)}, r_{t r}=\frac{\epsilon_{t r}^{u p}\left(t_{\infty}\right)}{\epsilon_{t r}^{\text {down }}\left(t_{\infty}\right)}$.
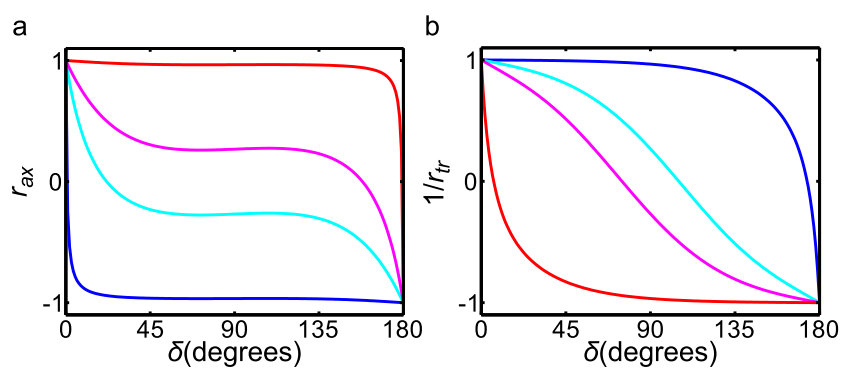

Fig. 5 Ratio of the steady-state strain in the top fiber to that in the bottom fiber in the longitudinal direction (a) and the transverse direction (b) as a function of the fiber alignment angle $\delta$ for force directions $\theta=1^{\circ}$ (blue), $\theta=30^{\circ}$ (cyan), $\theta=60^{\circ}$ (magenta) and $\theta=89^{\circ}$ (red)

Fig. 5 represents $r_{a x}$ and $r_{t r}^{-1}$ as a function of the fiber alignment angle $\delta$ for different force directions. For clarity, we plot $r_{t r}^{-1}$ instead of $r_{t r}$ to avoid infinite values and we restrict the representation to $\theta \in\left[0^{\circ}, 90^{\circ}\right]$. The symmetry of the system makes it straightforward to deduce the results for a broader range of $\theta$, i.e. $r(\theta+$ $180)=r(\theta)^{-1}$. A negative ratio value means that the top fiber is in compression while the bottom fiber is in tension. Fig. 5 shows that the mechanical signal in the top and bottom fibers can differ significantly $(|r|<<1)$. The strain in one fiber can be negligible compared to that in the other fiber $(|r| \approx 0)$, and one fiber can be in compression while the other is in tension $(r<0)$. Thus, force transmission can be strongly heterogeneous in space, the implications of which will be considered in the Discussion section.

The spatial heterogeneity in force transmission depends strongly on stress fiber alignment angle, $\delta$, and on force direction, $\theta$. Unless the fibers are perfectly aligned $\left(\delta=0^{\circ}\right)$, the strains are different in the two fibers and $|r| \neq 1$. We also note a plateau in the longitudinal strain ratio for intermediate values of $\theta$, so that over a broad range of stress fiber alignment angles $\delta$, the longitudinal strain ratio is independent of $\delta$ and depends only on force direction angle $\theta$. This plateau is absent in the case of the transverse strain ratio. Another significant difference between the transverse and longitudinal cases is the localization of the maximum strain. For the range of force directions $\theta$ represented in Fig. $5,\left|r_{a x}\right|<1$ so that the longitudinal strain in the top fiber is smaller than that in the bottom fiber. The opposite is true for the transverse strain.

3.5 Role of force profile: reversing vs. non-reversing forces

Experiments both in vivo and in vitro have shown that different shear stress profiles elicit different en- 
dothelial cell behavior. In particular, it has been suggested that atherosclerosis develops preferentially in zones of disturbed flow where the shear stress is low or reversing, whereas regions of high, non-reversing shear appear to be protected (Caro et al, 1969; Chatzizisis et al, 2007). To study possible differences in how forces characteristic of disturbed and undisturbed flow regions are transmitted, we consider an oscillatory nonreversing force $F_{A}$ and a reversing force $F_{B}$. These forces are defined by equation (18), where we choose $F_{0}=600 \mathrm{pN},\left(\alpha_{A}, \beta_{A}\right)=(0.5,0.75)$ and $\left(\alpha_{B}, \beta_{B}\right)=$ $(0.75,0.5)$. Thus, $\max \left(F_{A}\right)=\max \left(F_{B}\right)=375 \mathrm{pN}$, $\min \left(F_{A}\right)=75 \mathrm{pN}$ and $\min \left(F_{B}\right)=-75 \mathrm{pN}$, and the sign of $F_{A}$ does not change in time whereas the sign of $F_{B}$ does. Fig. 6a depicts the two applied force profiles over a period of oscillation $T$. We denote as $\epsilon_{A}$ and $\epsilon_{B}$ the norms of the fiber strains resulting from stimulation by the non-reversing force $F_{A}$ and by the reversing force $F_{B}$, respectively, and we plot the ratio of the maximum of $\epsilon_{B}$ to the maximum of $\epsilon_{A}$ over a period (Fig. 6b). Since the input forces $F_{A}$ and $F_{B}$ have the same maximum, a difference in the maximum of the resulting strains indicates a difference in signal transmission between the non-reversing and reversing cases. Interestingly, Fig. 6b shows that the ratio is always smaller than one, suggesting that a reversing force is less efficiently transmitted than a non-reversing force. When the fibers are isotropically organized $\left(\delta=90^{\circ}\right)$, the ratio does not depend on force direction and is small. As the fibers become more aligned, however, the ratio takes on a broader range of values depending on force direction. In the perfectly aligned configurations $\left(\delta=0^{\circ}\right.$ and $\delta=180^{\circ}$ ), the sensitivity to $\theta$ is maximum, and the ratio reaches its minimum if the force is applied along the fiber axis and its maximum (equal to one) if the force is applied transverse to the fibers. These observations hold for all frequencies tested, $0.1 \leq f \leq 10 \mathrm{~Hz}$. However, as frequency increases, the minimum value of the ratio decreases and the stress fibers need to be more aligned to allow the ratio to approach unity.

The results of Fig. 6 are related to the existence of the two time scales $\tau_{x}$ and $\tau_{y}$ introduced above. As previously discussed, the oscillatory part of the signal is cut off when its frequency is greater than the inverse of the characteristic time scale of the system. As for the constant part of the signal, its transmission does not depend on frequency. Since the reversing force has a smaller constant component $(\beta)$ and a larger oscillatory component $(\alpha)$, its transmission is more impacted by the cutting off of the oscillatory part of the signal than the non-reversing force. This cutting off of the oscillations occurs for configurations associated with a large time scale, i.e. for all force directions when the fiber
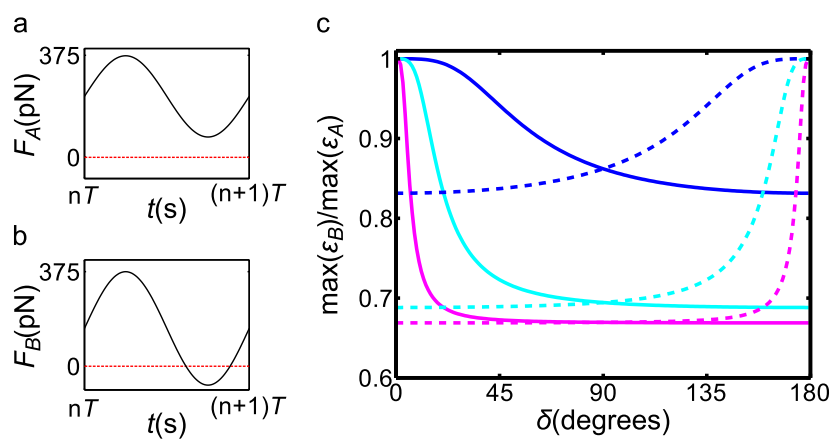

Fig. 6 Non-reversing (a) and reversing (b) force over a period $T$. c. Ratio of the maximum strain in the top fiber when a reversing force is applied to the maximum strain in the same fiber in response to a non-reversing force. The force is applied in the directions $\theta=0^{\circ}$ (solid lines) or $\theta=90^{\circ}$ (dashed lines) at a frequency of $f=0.1 \mathrm{~Hz}$ (blue), $f=1 \mathrm{~Hz}$ (cyan), and $f=10 \mathrm{~Hz}$ (magenta)

configuration is isotropic and for forces in the direction of the fibers when they are aligned. When $\beta$ decreases, the force becomes totally reversing and, in configurations associated with a characteristic time of deformation greater than the period of force oscillations, the stress fiber strain tends to zero.

\section{Discussion}

Consistent with previous experimental results $(\mathrm{Hu}$ and Wang, 2006; Hu et al, 2003; Na et al, 2008; Poh et al, 2009), the present model predicts that rapid longdistance force transmission depends centrally on stress fiber prestress. Stress fiber displacement in response to an externally applied force comparable to that used in previous experiments (Wang and Ingber, 1994) is found to be in good agreement with experimental results $(\mathrm{Hu}$ et al, 2003, 2004), $w=\epsilon L \sim 0.1 \mu \mathrm{m}$ (Fig. 3). The model also predicts the dynamics observed experimentally, in particular strain development within tens of milliseconds following application of a step force ( $\mathrm{Na}$ et al, 2008), as well as low-pass filter behavior ( $\mathrm{Hu}$ and Wang, 2006). Consistent with (Hu et al, 2004), the model predicts that in the case of an elongated morphology (aligned fibers), rapid dynamics are observed when forces are applied orthogonal to the stress fiber axis while forces exerted along the stress fiber axis are associated with slow dynamics (Fig. 4). Thus, the present model provides a theoretical framework that explains various experimental results. 
4.1 Prestressed stress fibers mediate rapid mechanical signal transmission

Experiments have shown that the application of a step force to integrins induces very rapid (within 300 msec) activation of the mechanosensitive protein Src at discrete intracellular sites as far away as $20 \mu \mathrm{m}$ from the site of force application ( $\mathrm{Na}$ et al, 2008). Intracellular diffusive transport and protein translocation via molecular motors would require several seconds to cover this distance, so these more traditional pathways for intracellular signaling fail to explain the experimental results ( $\mathrm{Na}$ et al, 2008). In contrast, our model predicts that the time scale for strain development in prestressed actin stress fibers ranges from a few milliseconds to a few seconds. For certain stress fiber configurations that are subjected to forces in particular directions, strains are transmitted within several hundred milliseconds (grey zones in Fig. 4), in line with the experimental observations on Src activation (Na et al, 2008). These findings are consistent with the hypothesis advanced in this paper that strain transmission is a mechanism for stress fiber-mediated mechanotransduction.

Interestingly, there are key limitations to this rapid strain transmission pathway. The present results indicate that only highly aligned stress fibers stimulated by a force sufficiently orthogonal to the direction of alignment of the fibers would allow strain transmission on this short time scale. In the arterial physiological context of an oscillatory force of period $T$, this implies that a force sufficiently orthogonal to the fibers is efficiently transmitted within a cell even for high oscillation frequencies on the order of a kilohertz, whereas a force along the fibers is cut off when the oscillation frequency exceeds $0.1 \mathrm{~Hz}$. This observation is in agreement with experimental results ( $\mathrm{Hu}$ et al, 2004; $\mathrm{Hu}$ and Wang, 2006).

An interesting prediction of the model is that the definition of a sufficiently orthogonal forcing is very strict in the case of a longitudinal strain, as a force only a few degrees away from the orthogonal fails to induce rapid longitudinal strain. On the other hand, a force at an angle of up to $50^{\circ}$ away from the orthogonal direction can still elicit rapid transverse strain. The very narrow range of conditions allowing rapid longitudinal strain suggests that downstream signaling events that need to be robustly rapid would need to rely on transverse rather than longitudinal strain of the fibers.

The model predictions can, in principle, be tested experimentally by culturing cells on patterned surfaces that allow control of cytoskeletal organization and subsequently subjecting the cells to oscillating forces at controlled directions and frquencies.
4.2 Stress fibers: a critical link in the mechanotransduction chain?

Over the past years, many studies have focused on understanding how cells sense and respond to mechanical forces. An emerging paradigm is that mechanical forces change the chemical landscape of the cell either by altering intracellular reaction kinetics, uncovering cryptic binding sites, or bringing together molecules that would otherwise be apart (Hoffman et al, 2011; Vogel, 2006; Janmey, 1998). In particular, several proteins that localize to focal adhesions including p130Cas (Sawada et al, 2006), zyxin (Lele et al, 2006) or talin (del Rio et al, 2009) have been shown to change their activity under force. Some of these proteins have also been shown to link to stress fibers in a manner dependent upon stress fiber stretch, and it has been suggested that protein binding affinity to stress fibers is altered by tension (Sawada and Sheetz, 2002; Yoshigi et al, 2005; Colombelli et al, 2009). Our model indicates that the force-induced strain in stress fibers is not localized to the portion of the stress fiber in contact with focal adhesions but is rather distributed throughout the length of the stress fiber. Thus, we propose that mechano-chemical conversion may occur anywhere along the stress fiber length and not only at focal adhesions. This prediction is supported by recent experiments showing activation of the protein c-Src along stress fibers through binding to the mechanosensitive protein AFAP (Han et al, 2004). Activation of Src following application of a stress of $20 \mathrm{~Pa}$ by magnetic tweezers (Na et al, 2008) shows that the level of mechanical strain predicted by our model is sufficient to elicit such biological response. A mechanism that explains stress fiber strain perception by proteins has been suggested for zyxin. Zyxin, which has been implicated in the stabilization of stress fibers (Smith et al, 2010), has several LIM domains, which may act as a ruler to measure the distance between binding sites (Schiller and Fässler, 2013), so that the extent of zyxin binding to a stress fiber would be directly related to the strain in the stress fiber.

The present results show the potential richness of the stress fiber mechano-transmission pathway. In our model, the extent (Fig. 3) and dynamics (Fig. 4) of strain that a protein linked to a stress fiber would experience depend on the direction of the external force, the extent of stress fiber alignment, and the way the protein binds to the stress fiber, since whether a protein undergoes transverse or longitudinal strain depends on how the protein is attached to the fiber. If the protein activity increases with stress fiber longitudinal strain, then its average activity would be greater in aligned 
stress fiber networks subjected to force in the direction of alignment, a configuration characteristic of atheroprotected regions, than in isotropic stress fiber networks subjected to low, direction-changing, or reversing forces (Fig. 3a). The opposite would be true if the protein were responsive to transverse strain (Fig. 3b). These examples show how the biological activity of a protein that is sensitive to stress fiber strain can potentially be modulated by mechanical cues applied at the cell membrane and how different proteins that have different functions can be modulated differently.

The fact that the dynamics of strain development in stress fibers can range from milliseconds to several seconds depending on stress fiber organization and on force direction (Fig. 4) may provide cells with the ability for temporal orchestration of responses to mechanical stimulation. In endothelial cells, relatively rapid forceinduced responses including activation of mechanosensitive ion channels and of integrins as well as mobilization of intracellular calcium have been shown to occur over time scales ranging from a fraction of a second to several seconds after the onset of the mechanical stimulus (Kholodenko et al, 2010). These time scales are consistent with the results of the present model.

In considering stresses and strains in stress fibers, it is useful to think about the localization of stress fibers within cells. There is ample evidence that stress fibers are present in the basal regions of cells where they connect focal adhesion sites. In the case of endothelial cells in vivo, basal stress fibers would not experience blood flow-derived forces directly but would rather be subjected to stretch due to the periodic circumferential expansion of compliant blood vessels. Thus, unlike the situation considered in the current model, basal stress fibers are expected to be subjected to external strains rather than external forces. On the other hand, there is evidence that in various cell types including endothelial cells, the apical cell surface exhibits focal adhesion-type structures that have been labeled "apical plaques" and that connect apical stress fibers (Conforti et al, 1992; Kano et al, 1996; Katoh et al, 2008). Blood flow would directly subject these apical stress fibers to external forces as formulated in the present paper.

\subsection{Cell polarization under force: the strain track}

A prominent response of endothelial cells to shear stress is cellular polarization and alignment in the direction of shear (Dewey et al, 1981; Helmlinger et al, 1991). Several mechanisms have been proposed to explain this polarization, including shear rate-dependent gradients of chemical cues around the cell (Shamloo et al, 2008) and shear stress-dependent activation of small GTPases at focal adhesions that triggers cytoskeletal remodeling (Shyy and Chien, 2002; Li et al, 1999). Our model shows that an external force induces a spatially heterogeneous strain in the stress fiber network (Fig. 5). As discussed above, this can in turn induce directional heterogeneity of protein activation. Since this heterogeneous strain contains information on force directionality, we propose stress fiber strain as a candidate mechanism through which a force can trigger early events in cell polarization.

4.4 Low-level stress fiber strain as a key feature of disturbed flow regions

It has been experimentally observed that cells respond differently to different types of forces. For instance, endothelial cells subjected to high, nonreversing shear stress exhibit a stress fiber architecture that is aligned in the direction of the applied force, and these cells exhibit a quiescent, anti-inflammatory, and atheroprotective phenotype. In contrast, cells subjected to low or reversing shear stress adopt an isotropic stress fiber organization and express an inflammatory phenotype that favors the development of atherosclerosis (Chatzizisis et al, 2007; Malek et al, 1999; Hahn and Schwartz, 2009). Our results demonstrate that a reversing force elicits smaller stress fiber strain than a non-reversing force (Fig. 6). At physiological frequencies, the strain difference can be as large as 30\% even for the mild reversal considered in Fig. 6. Our model also predicts that a low non-reversing shear would lead to a small strain of the stress fibers. Thus, our results indicate that low-level stress fiber strain is a common feature of both reversing and low shear flows. As discussed above, this may impact the activity of many proteins and subsequent signaling pathways and may play a role in the cell's adoption of an atheroprone or atheroprotective phenotype.

4.5 The two-fiber system is representative of more complex stress fiber networks

To investigate how representative the simple twofiber system studied above is representative of more complex stress fiber networks, we study strain transmission through a network of four stress fibers of identical mechanical properties as represented in Fig. 7a. The four stress fibers link a membrane protein (moving node $M$ in Fig. 7a) to distinct intracellular sites (fixed nodes $M_{1}, M_{2}, M_{3}$ and $M_{4}$ in Fig. 7a). Extension of the analysis described for the two-fiber system (equations (9) to (12)) yields ordinary differential equations 
governing the displacement of the moving node:

$\boldsymbol{\Gamma} \frac{d \mathbf{w}_{M}}{d t}=-\mathbf{K w}_{M}+\mathbf{F}_{e x t}$,

where $\mathbf{K}$ and $\boldsymbol{\Gamma}$ are respectively the stiffness and damping matrices:

$$
\begin{aligned}
& \mathbf{K}= \\
& \sum_{i=1}^{4}\left[\begin{array}{ll}
k_{v} \sin ^{2}\left(\delta_{i}\right)+k_{l} \cos ^{2}\left(\delta_{i}\right) & \left(k_{l}-k_{v}\right) \sin \left(\delta_{i}\right) \cos \left(\delta_{i}\right) \\
\left(k_{l}-k_{v}\right) \sin \left(\delta_{i}\right) \cos \left(\delta_{i}\right) & k_{v} \cos ^{2}\left(\delta_{i}\right)+k_{l} \sin ^{2}\left(\delta_{i}\right)
\end{array}\right],
\end{aligned}
$$

$$
\begin{aligned}
& \boldsymbol{\Gamma}= \\
& \sum_{i=1}^{4}\left[\begin{array}{ll}
\gamma_{v} \sin ^{2}\left(\delta_{i}\right)+\gamma_{l} \cos ^{2}\left(\delta_{i}\right) & \left(\gamma_{l}-\gamma_{v}\right) \sin \left(\delta_{i}\right) \cos \left(\delta_{i}\right) \\
\left(\gamma_{l}-\gamma_{v}\right) \sin \left(\delta_{i}\right) \cos \left(\delta_{i}\right) & \gamma_{v} \cos ^{2}\left(\delta_{i}\right)+\gamma_{l} \sin ^{2}\left(\delta_{i}\right)
\end{array}\right],
\end{aligned}
$$

where $k_{l}=\frac{E A}{L}, k_{v}=\frac{\sigma_{p} A}{L}, \gamma_{l}=\frac{\gamma A}{L}, \gamma_{v}=\frac{\gamma I}{L^{3}}$, and $\delta_{i}$ is the angle between the $\mathrm{x}$-axis and the fiber $f_{i}$.

Random four-fiber configurations are generated by choosing random values of the angles $\delta_{i}$. The isotropy of the network is assessed by computing the isotropy index $q$, defined as the mean angular distance between a fiber and the mean fiber direction:

$q=\frac{<\left|\tilde{\delta}_{i}-<\tilde{\delta}_{i}>_{i \in[1,4]}\right|>_{i \in[1,4]}}{45}$,

where $\tilde{\delta}_{i}+n_{i} \times 180^{\circ}$, with $n_{i}=0$ or $n_{i}=1$. The values of $n_{i}$ are chosen so that all $\tilde{\delta}_{i}$ lie in an interval of length $180^{\circ}$. If the four fibers are perfectly aligned, $q=0$, whereas in the isotropic case where the angle between two fibers is $90^{\circ}, q=1$. Note that this is also true for the system of two fibers.

We define the characteristic stiffnesses, $K_{1}$ and $K_{2}$, and the characteristic times, $\tau_{1}$ and $\tau_{2}$, associated with the deformation of the two- or four-fiber network as the eigenvalues of the stiffness matrix $K$ (equations (11b) and (18b)) and of $K^{-1} \Gamma$ where $\Gamma$ is the damping matrix (equations (11c) and (18c)). Figs. 7b and c show respectively the characteristic stiffnesses and the characteristic times of the four-fiber network stiffness matrix (dots in Figs. 7b and c) and of the two-fiber system (equation (solid line in Figs. 7b and c). The results for the fourfiber system are noisy because several four-fiber configurations correspond to a given isotropy index. However, the noise is small compared to the dependence of the results on the isotropy index. This dependence is very well fitted by the results obtained for the two-fiber system. The magnitude of the characteristic stiffnesses is the only major difference between the two-fiber and the a
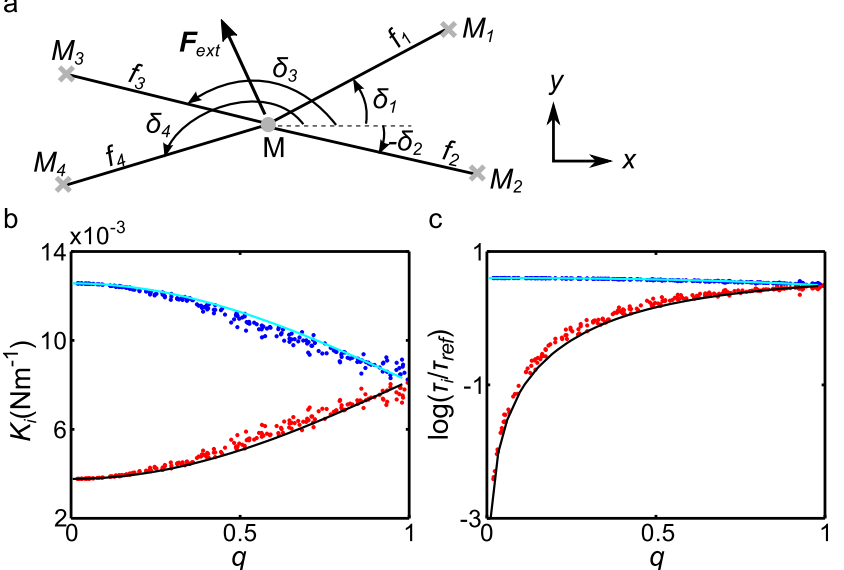

Fig. 7 a. Schematic of a network of four fibers linking a membrane protein (moving node $M$ ) to intracellular structures (fixed nodes $M_{i}$ ). b. Four-fiber network characteristic stiffnesses $K_{1}$ and $K_{2}$ (blue and red dots respectively) and twofiber network characteristic stiffnesses divided by two (cyan and black solid lines) as a function of the isotropy index $q$. c. Four-fiber network (blue and red dots) and two-fiber network characteristic times of deformation $\tau_{1}$ and $\tau_{2}$ as a function of the isotropy index $q \cdot \tau_{r e f}=1 \mathrm{sec}$

four-fiber systems. Indeed, more fibers resist the system deformation in the four-fiber network; consequently, the characteristic stiffnesses are twice those of the two-fiber system.

Given that the characteristics of strain transmission are driven by the eigenvalues of the stiffness and damping matrices, the consistency of the dependence of characteristic times and stiffnesses on stress fiber organization shows that the results of the two-fiber model can indeed be used to characterize the behavior of more complex networks.

\section{Conclusions}

We have developed a model to study the transmission of mechanical signals in a network of prestressed viscoelastic actin stress fibers. To understand the correlation between external force characteristics, stress fiber alignment, and expression of atheroprotective or atheroprone genes, we studied simple systems of fibers whose unique parameters are the alignment of the fibers and the external force characteristics. We showed that the dynamics of force transmission in the fibers are consistent with experimental results obtained by applying forces to cells using magnetic tweezers, and we proposed that strain-dependent binding of proteins to stress fibers may explain cell polarization and differences in cell function in disturbed versus undisturbed flow regions. We thus propose that stress fiber strain may be an intermediate mechanism to translate a force 
signal applied to the cell into a chemical signal, via the activation of strain-dependent proteins within the cell.

Although the networks considered in the present work are very simple, we expect them to capture the essential features of mechanical signal transmission through more complex stress fiber networks. In fact, the theoretical framework developed here can be readily expanded to allow the study of two- and threedimensional networks of arbitrary complexity. Such a study, however, awaits quantitative experimental data on the topology of stress fiber networks in cells.

Acknowledgements CLM Gouget is supported by Ecole Polytechnique through a Gaspard Monge International Scholarship. This work was funded in part by an endowment in Cardiovascular Cellular Engineering from the AXA Research Fund.

\section{References}

Ashkin A, Schütze K, Dziedzic J, Euteneuer U, Schliwa M (1990) Force generation of organelle transport measured in vivo by an infrared laser trap. Nature 348:346-348

Barakat AI, Lieu DK, Gojova A (2006) Secrets of the code: do vascular endothelial cells use ion channels to decipher complex flow signals? Biomaterials 27(5):671-678

Blumenfeld R (2006) Isostaticity and controlled force transmission in the cytoskeleton: a model awaiting experimental evidence. Biophysical journal 91(5):1970-1983

Caro C, Fitz-Gerald J, Schroter R (1969) Arterial wall shear and distribution of early atheroma in man. Nature 223:1159-1161

Chatzizisis YS, Coskun AU, Jonas M, Edelman ER, Feldman CL, Stone PH (2007) Role of endothelial shear stress in the natural history of coronary atherosclerosis and vascular remodeling. J Am Coll Cardiol 49(25):2379-2393

Chien S (2007) Mechanotransduction and endothelial cell homeostasis: the wisdom of the cell. American Journal of Physiology-Heart and Circulatory Physiology 292(3):H1209-H1224

Choquet D, Felsenfeld DP, Sheetz MP (1997) Extracellular matrix rigidity causes strengthening of integrincytoskeleton linkages. Cell 88(1):39-48

Colombelli J, Besser A, Kress H, Reynaud EG, Girard P, Caussinus E, Haselmann U, Small JV, Schwarz US, Stelzer EH (2009) Mechanosensing in actin stress fibers revealed by a close correlation between force and protein localization. J Cell Sci 122(10):1665-1679
Conforti G, Dominguez-Jimenez C, Zanetti A, Gimbrone Jr MA, Cremona O, Marchisio P, Dejana E (1992) Human endothelial cells express integrin receptors on the luminal aspect of their membrane. Blood 80(2):437-446

Costa M, Marchi M, Cardarelli F, Roy A, Beltram F, Maffei L, Ratto GM (2006) Dynamic regulation of erk2 nuclear translocation and mobility in living cells. J Cell Sci 119(23):4952-4963

Davies PF (2008) Hemodynamic shear stress and the endothelium in cardiovascular pathophysiology. Nat Clin Pract Cardiovasc Med 6(1):16-26

Davies PF, Robotewskyj A, Griem M (1993) Endothelial cell adhesion in real time. measurements in vitro by tandem scanning confocal image analysis. J Clin Invest 91(6):2640

Davies PF, Robotewskyj A, Griem ML (1994) Quantitative studies of endothelial cell adhesion. directional remodeling of focal adhesion sites in response to flow forces. J Clin Invest 93(5):2031

Deguchi S, Ohashi T, Sato M (2006) Tensile properties of single stress fibers isolated from cultured vascular smooth muscle cells. J Biomech 39(14):2603-2610

Dewey C, Gimbrone M, Davies P, Bussolari S (1981) The dynamic response of vascular endothelial cells to fluid shear stress. J Biomech Eng 103(3):177-185

Flaherty JT, Pierce JE, Ferrans VJ, Patel DJ, Tucker WK, Fry DL (1972) Endothelial nuclear patterns in the canine arterial tree with particular reference to hemodynamic events. Circ Res 30(1):23-33

Florian JA, Kosky JR, Ainslie K, Pang Z, Dull RO, Tarbell JM (2003) Heparan sulfate proteoglycan is a mechanosensor on endothelial cells. Circ Res 93(10):e136-e142

Friedland JC, Lee MH, Boettiger D (2009) Mechanically activated integrin switch controls $\alpha 5 \beta 1$ function. Science 323(5914):642-644

Galbraith C, Skalak R, Chien S (1998) Shear stress induces spatial reorganization of the endothelial cell cytoskeleton. Cell Motil and the Cytoskel 40(4):317330

Geiger B, Spatz JP, Bershadsky AD (2009) Environmental sensing through focal adhesions. Nat Rev Mol Cell Biol 10(1):21-33

Hahn C, Schwartz MA (2009) Mechanotransduction in vascular physiology and atherogenesis. Nat Rev Mol Cell Biol 10(1):53-62

Haidekker MA, L'Heureux N, Frangos JA (2000) Fluid shear stress increases membrane fluidity in endothelial cells: a study with dcvj fluorescence. Am J Physiol-Heart Circ Physiol 278(4):H1401-H1406

Han B, Bai XH, Lodyga M, Xu J, Yang BB, Keshavjee S, Post M, Liu M (2004) Conversion of mechan- 
ical force into biochemical signaling. J Biol Chem 279(52):54,793-54,801

Helmlinger G, Geiger R, Schreck S, Nerem R (1991) Effects of pulsatile flow on cultured vascular endothelial cell morphology. J Biomech Eng 113(2):123-131

Hoffman BD, Grashoff C, Schwartz MA (2011) Dynamic molecular processes mediate cellular mechanotransduction. Nature 475(7356):316-323

$\mathrm{Hu}$ S, Wang N (2006) Control of stress propagation in the cytoplasm by prestress and loading frequency. Mol Cell Biomech 3(2):49

Hu S, Chen J, Fabry B, Numaguchi Y, Gouldstone A, Ingber DE, Fredberg JJ, Butler JP, Wang N (2003) Intracellular stress tomography reveals stress focusing and structural anisotropy in cytoskeleton of living cells. Am J Physiol-Cell Physiol 285(5):C1082-C1090

$\mathrm{Hu} \mathrm{S}$, Eberhard L, Chen J, Love JC, Butler JP, Fredberg JJ, Whitesides GM, Wang N (2004) Mechanical anisotropy of adherent cells probed by a three-dimensional magnetic twisting device. Am J of Physiol-Cell Physiol 287(5):C1184-C1191

Hu S, Chen J, Butler JP, Wang N (2005) Prestress mediates force propagation into the nucleus. Biochem Biophys Res Commun 329(2):423-428

Hwang Y, Barakat AI (2012) Dynamics of mechanical signal transmission through prestressed stress fibers. PloS One 7(4):e35,343

Hwang Y, Gouget CLM, Barakat AI (2012) Mechanisms of cytoskeleton-mediated mechanical signal transmission in cells. Comm Int Biol 5(6):538-542

Janmey PA (1998) The cytoskeleton and cell signaling: component localization and mechanical coupling. Physiol Rev 78(3):763-781

Kano Y, Katoh K, Masuda M, Fujiwara K (1996) Macromolecular composition of stress fiber-plasma membrane attachment sites in endothelial cells in situ. Circulation research 79(5):1000-1006

Katoh K, Kano Y, Ookawara S (2008) Role of stress fibers and focal adhesions as a mediator for mechanosignal transduction in endothelial cells in situ. Vascular health and risk management 4(6):1273

Kholodenko BN, Hancock JF, Kolch W (2010) Signalling ballet in space and time. Nat Rev Mol Cell Biol 11(6):414-426

Kumar S, Maxwell IZ, Heisterkamp A, Polte TR, Lele TP, Salanga M, Mazur E, Ingber DE (2006) Viscoelastic retraction of single living stress fibers and its impact on cell shape, cytoskeletal organization, and extracellular matrix mechanics. Biophys J 90(10):3762-3773

Leckband DE, le Duc Q, Wang N, de Rooij J (2011) Mechanotransduction at cadherin-mediated adhesions. Curr Opin Cell Biol 23(5):523-530
Lele TP, Pendse J, Kumar S, Salanga M, Karavitis J, Ingber DE (2006) Mechanical forces alter zyxin unbinding kinetics within focal adhesions of living cells. J Cell Physiol 207(1):187-194

Li S, Chen BP, Azuma N, Hu YL, Wu SZ, Sumpio BE, Shyy JYJ, Chien S (1999) Distinct roles for the small gtpases cdc42 and rho in endothelial responses to shear stress. J Clin Invest 103(8):1141-1150

Lu L, Oswald SJ, Ngu H, Yin FCP (2008) Mechanical properties of actin stress fibers in living cells. Biophys J 95(12):6060-6071

Malek AM, Alper SL, Izumo S (1999) Hemodynamic shear stress and its role in atherosclerosis. J Am Med Assoc 282(21):2035-2042

Na S, Collin O, Chowdhury F, Tay B, Ouyang M, Wang Y, Wang N (2008) Rapid signal transduction in living cells is a unique feature of mechanotransduction. Proc Natl Acad Sci 105(18):6626-6631

Orr AW, Helmke BP, Blackman BR, Schwartz MA (2006) Mechanisms of mechanotransduction. Dev Cell 10(1):11-20

Poh YC, Na S, Chowdhury F, Ouyang M, Wang Y, Wang N (2009) Rapid activation of rac gtpase in living cells by force is independent of src. PLoS One 4(11):e7886

Poh YC, Shevtsov SP, Chowdhury F, Wu DC, Na S, Dundr M, Wang N (2012) Dynamic force-induced direct dissociation of protein complexes in a nuclear body in living cells. Nature communications 3:866

del Rio A, Perez-Jimenez R, Liu R, Roca-Cusachs P, Fernandez JM, Sheetz MP (2009) Stretching single talin rod molecules activates vinculin binding. Science 323(5914):638-641

Sawada Y, Sheetz MP (2002) Force transduction by triton cytoskeletons. J Cell Biol 156(4):609-615

Sawada Y, Tamada M, Dubin-Thaler BJ, Cherniavskaya O, Sakai R, Tanaka S, Sheetz MP (2006) Force sensing by mechanical extension of the src family kinase substrate p130cas. Cell 127(5):1015-1026

Schiller HB, Fässler R (2013) Mechanosensitivity and compositional dynamics of cell-matrix adhesions. EMBO Rep 14(6):509-519

Shamloo A, Ma N, Poo Mm, Sohn LL, Heilshorn SC (2008) Endothelial cell polarization and chemotaxis in a microfluidic device. Lab Chip 8(8):1292-1299

Shyy JYJ, Chien S (2002) Role of integrins in endothelial mechanosensing of shear stress. Circ Res 91(9):769-775

Smith MA, Blankman E, Gardel ML, Luettjohann L, Waterman CM, Beckerle MC (2010) A zyxinmediated mechanism for actin stress fiber maintenance and repair. Dev cell 19(3):365-376 
Sukharev S, Betanzos M, Chiang CS, Guy HR (2001) The gating mechanism of the large mechanosensitive channel mscl. Nature 409(6821):720-724

Tarbell JM, Pahakis M (2006) Mechanotransduction and the glycocalyx. J Intern Med 259(4):339-350

Tzima E, Irani-Tehrani M, Kiosses WB, Dejana E, Schultz DA, Engelhardt B, Cao G, DeLisser H, Schwartz MA (2005) A mechanosensory complex that mediates the endothelial cell response to fluid shear stress. Nature 437(7057):426-431

Vogel V (2006) Mechanotransduction involving multimodular proteins: converting force into biochemical signals. Annu Rev Biophys Biomol Struct 35:459-488

Wang N, Ingber DE (1994) Control of cytoskeletal mechanics by extracellular matrix, cell shape, and mechanical tension. Biophys J 66(6):2181-2189

Wang N, Suo Z (2005) Long-distance propagation of forces in a cell. Biochemical and biophysical research communications 328(4):1133-1138

Wang N, Tytell JD, Ingber DE (2009) Mechanotransduction at a distance: mechanically coupling the extracellular matrix with the nucleus. Nat Rev Mol Cell Biol 10(1):75-82

Wong AJ, Pollard TD, Herman IM (1983) Actin filament stress fibers in vascular endothelial cells in vivo. Science 219(4586):867-869

Yonemura S, Wada Y, Watanabe T, Nagafuchi A, Shibata M (2010) $\alpha$-catenin as a tension transducer that induces adherens junction development. Nat Cell Biol 12(6):533-542

Yoshigi M, Hoffman LM, Jensen CC, Yost HJ, Beckerle MC (2005) Mechanical force mobilizes zyxin from focal adhesions to actin filaments and regulates cytoskeletal reinforcement. J Cell Biol 171(2):209-215

\section{Appendix}

Results obtained with a single stress fiber (Hwang and Barakat, 2012) suggest that stress fiber inertia is negligible, so that wave perturbations in the deformation field are damped by fiber internal viscosity. In support of this notion, the results show that force transmission dynamics are indeed dominated by spatially monotonic deformation of stress fibers. Therefore, the structure of the deformation field does not change significantly in time, and displacement of the fiber can be written as:

$w_{v}(x, t)=a_{v}(t) \psi_{v}(x)$,

$w_{l}(x, t)=a_{l}(t) \psi_{l}(x)$
Substituting equations (20a) and (20b) into (1a) and (1b) and integrating these equations over the spatial domain yields:

$$
\begin{aligned}
& \frac{\sigma_{p} A}{L} \underbrace{\left(\int_{0}^{1} \frac{d^{2} \psi_{v}(\hat{x})}{d \hat{x}^{2}} d \hat{x}\right)}_{C_{1, v}} a_{v}(t)+ \\
& \frac{\gamma I}{L^{3}} \underbrace{\left(-\int_{0}^{1} \frac{d^{4} \psi_{v}(\hat{x})}{d \hat{x}^{4}} d \hat{x}\right)}_{C_{2, v}} \frac{d a_{v}(t)}{d t}+F_{v}=0,
\end{aligned}
$$

$\frac{E A}{L} \underbrace{\left(\int_{0}^{1} \frac{d^{2} \psi_{l}(\hat{x})}{d \hat{x}^{2}} d \hat{x}\right)}_{C_{l}} a_{l}(t)+$

$\frac{\gamma A}{L} \underbrace{\left(\int_{0}^{1} \frac{d^{2} \psi_{l}(\hat{x})}{d \hat{x}^{2}} d \hat{x}\right)}_{C_{l}} \frac{d a_{l}(t)}{d t}+F_{l}=0$

where $\hat{x}$ is defined as $x / L$.

Equations (20a) and (20b) can be used to relate the displacement of the free end of the fiber to the time functions $a_{v}$ and $a_{l}$ : $w_{v}^{\text {end }}(t)=a_{v}(t) \psi_{v}(0)$ and $w_{l}^{e n d}(t)=a_{l}(t) \psi_{l}(0)$ and $w_{l}(t)=a_{l}(t) \psi_{l}(0)$. Rearranging equations $(21)$ with $\hat{C}_{1, v}=C_{1, v} / \psi_{v}(0), \hat{C}_{2, v}=$ $C_{2, v} / \psi_{v}(0)$ and $\hat{C}_{l}=C_{l} / \psi_{l}(0)$, we obtain the following ordinary differential equations (ODEs) that describe the motion of the free end of the fiber $(x=0)$ :

$\frac{\sigma_{p} A}{L} \hat{C}_{1, v} w_{v}^{e n d}(t)+\frac{\gamma I}{L^{3}} \hat{C}_{2, v} \frac{d w_{v}^{e n d}(t)}{d t}+F_{v}=0$

$\frac{E A}{L} \hat{C}_{l} w_{l}^{e n d}(t)+\frac{\gamma A}{L} \hat{C}_{l} \frac{d w_{l}^{e n d}(t)}{d t}+F_{l}=0$.

An order of magnitude analysis on the three constants $\hat{C}_{1, v}, \hat{C}_{2, v}$ and $\hat{C}_{l}$ reveals that their magnitudes are $O(1)$. We detail the analysis for the case of $\hat{C}_{1, v}$ :

$\hat{C}_{1, v}=\frac{1}{\psi_{v}(0)} \int_{0}^{1} \frac{d^{2} \psi_{v}(\hat{x})}{d \hat{x}^{2}} d \hat{x}=\left.\frac{1}{\psi_{v}(0)} \frac{d \psi_{v}(\hat{x})}{d \hat{x}}\right|_{\hat{x}=1}$,

given that the boundary condition at $x=0$ imposes that $d \psi_{v}(\hat{x}) /\left.d \hat{x}\right|_{\hat{x}=0}=0$. The derivative of $\psi_{v}$ at $\hat{x}=1$ can be approximated by $\left(\psi_{v}(1)-\psi_{v}(0)\right) /(1-0)$, where $\psi_{v}(1)=0$. Substituting this into equation 23 yields $\hat{C}_{1, v}=O(1)$.

Because forces associated with prestress, elasticity, and material viscosity act against the direction of the externally applied force, their signs should be negative, and it is reasonable to approximate $\hat{C}_{1, v}=\hat{C}_{2, v}=\hat{C}_{l}=$ -1 . Hence, the transverse and longitudinal motions of the free end are governed by the two ODEs given by equations $5 \mathrm{a}$ and $5 \mathrm{~b}$. 